\title{
The PIN gene family in cotton (Gossypium hirsutum): genome-wide identification and gene expression analyses during root development and abiotic stress responses
}

\author{
Peng He ${ }^{1,2+}$, Peng Zhao ${ }^{1 \dagger}$, Limin Wang ${ }^{3+}$, Yuzhou Zhang ${ }^{2}$, Xiaosi Wang ${ }^{2}$, Hui Xiao ${ }^{2}$, Jianing Yu ${ }^{2^{*}}$
} and Guanghui Xiao ${ }^{1 *}$

\begin{abstract}
Background: Cell elongation and expansion are significant contributors to plant growth and morphogenesis, and are often regulated by environmental cues and endogenous hormones. Auxin is one of the most important phytohormones involved in the regulation of plant growth and development and plays key roles in plant cell expansion and elongation. Cotton fiber cells are a model system for studying cell elongation due to their large size. Cotton is also the world's most utilized crop for the production of natural fibers for textile and garment industries, and targeted expression of the IAA biosynthetic gene iaaM increased cotton fiber initiation. Polar auxin transport, mediated by PIN and AUX/LAX proteins, plays a central role in the control of auxin distribution. However, very limited information about PIN-FORMED (PIN) efflux carriers in cotton is known.

Results: In this study, 17 PIN-FORMED (PIN) efflux carrier family members were identified in the Gossypium hirsutum (G. hirsutum) genome. We found that PIN1-3 and PIN2 genes originated from the At subgenome were highly expressed in roots. Additionally, evaluation of gene expression patterns indicated that PIN genes are differentially induced by various abiotic stresses. Furthermore, we found that the majority of cotton PIN genes contained auxin (AuxREs) and salicylic acid (SA) responsive elements in their promoter regions were significantly up-regulated by exogenous hormone treatment.

Conclusions: Our results provide a comprehensive analysis of the PIN gene family in G. hirsutum, including phylogenetic relationships, chromosomal locations, and gene expression and gene duplication analyses. This study sheds light on the precise roles of PIN genes in cotton root development and in adaption to stress responses.
\end{abstract}

Keywords: Auxin, PIN-formed, Cotton, Root development, Expression patterns, Abiotic stress

\section{Background}

The plant phytohormone auxin (indole-3-acetic acid, IAA) plays an essential role in plant morphogenesis, organogenesis, apical dominance, embryo formation, vascular differentiation, and light and gravity perception

\footnotetext{
* Correspondence: jnyu@snnu.edu.cn; guanghuix@snnu.edu.cn

${ }^{\dagger}$ Equal contributors

${ }^{2}$ College of Life Sciences, Shaanxi Normal University, Xi'an 710119, China ${ }^{1}$ Key Laboratory of the Ministry of Education for Medicinal Plant Resources and Natural Pharmaceutical Chemistry, National Engineering Laboratory for Resource Development of Endangered Crude Drugs in the Northwest of China, College of Life Sciences, Shaanxi Normal University, Xi'an 710119, China

Full list of author information is available at the end of the article
}

$[1,2]$. Two critical pathways, including auxin transport and auxin signaling, are vital for plant development, playing a major role in both phototropism and gravitropism. At the cellular level, auxin negatively regulates the transcription factor AUXIN RESPONSE FACTOR (ARF), which mediates the expression of auxin-responsive genes $[3,4]$. At the tissue level, auxin is synthesized and then transported from the site of biosynthesis to the sites of auxin action [5, 6]. Auxin efflux has been observed in specific tissues at different developmental stages, and plays a role in lateral organ initiation, root gravitropism, and root hair formation [7-9]. The cell to cell polar transport 
of auxin is mediated by specific influx and efflux carriers, resulting in asymmetric distribution of auxin $[10,11]$. The auxin influx and efflux proteins in Arabidopsis can be grouped into 3 gene families: P-glycoprotein (MDR/PGP/ $\mathrm{ABCB}$ ) efflux/conditional transporters (PGP), auxin resistant 1/like aux1 (AUX1/LAX) influx carriers, and plant specific PIN-FORMED (PIN) efflux carriers $[4,12,13]$. The polar subcellular localization of PIN efflux proteins is responsible for directional auxin flow, and more and more evidence indicates that plasma membrane-localized PIN proteins are the rate-limiting step in auxin polar transport $[14,15]$. PIN polarization and auxin polar transport play an important role in both plant phototropism and gravitropism [16, 17].

In Arabidopsis, the PIN gene family is comprised of 8 members, which have been reported to be involved in various developmental processes [18, 19]. PIN1, PIN2, PIN3, PIN14 and PIN7, located in the plasma membrane, are involved in the tropic response and root growth. In contrast, the endoplasmic reticulum (ER) localized PINs, including PIN5, PIN6 and PIN8, are mainly involved in intracellular auxin homeostasis [20, 21]. Among all PIN family members, loss-of-function pin1 and pin 2 mutants show severe phenotypes, making them well suited for investigating auxin-dependent developmental processes [22]. PIN1 is mainly involved in the maintenance of embryonic auxin gradients, and organ initiation is severely affected in pin1 mutants, which results in the formation of naked inflorescence stems [23]. Constitutive triple response 1 (CTR1), a protein that acts downstream of the ethylene receptors, is a negative regulator of ethylene signaling. PIN2 functions downstream of CTR1 and primarily regulates root gravitropism [24]. The roots of pin2 mutants are insensitive to ethylene and grow agravitropically $[25,26]$. Recently, the PIN genes have been reported to play a role in the integration of hormone signaling and abiotic stress responses. In soybean, PIN genes are differentially regulated by both abiotic stresses and phytohormones [19]. In rice, PIN genes show tissuespecific expression and have been found to also be regulated by hormones [27]. In maize, the expression levels of most ZmPIN genes were induced in shoots and reduced in roots by various abiotic stress treatments, including drought, salt, dehydration and cold [15].

Cotton is an important source of both natural fibers in the textile industry and cotton seed oil used in the production of food and biodiesel fuel. Understanding the factors that regulate fiber initiation will drive the development of technologies to improve yield potentials. Prior work suggests that auxin plays an essential role in fiber cell initiation. Exogenous application of IAA to cotton ovules promoted cotton fiber initials during fiber cell initiation [28]. Furthermore, in vitro application of N-1-naphthylphtha-lamic acid (NPA), an inhibitor of auxin polar transport, reduces IAA accumulation and inhibits fiber cell initiation [29]. Further, targeted expression of iaaM, an IAA biosynthetic gene, was showed to increase the number of lint fibers produced [29]. In additional, auxin regulates cotton fiber initiation via GhPIN-mediated auxin transport [30,31]. Auxin is not synthesized in fiber cells, and is mainly transported from the outside of ovules to fiber cells via polar auxin transport.

In this work, we performed a comprehensive analysis of the PIN gene family based on data gathered from recent whole genome sequencing results [32-36]. Comparative analysis of allotetraploid cotton (G. hirsutum) with its diploid ancestor (G. arboreum) indicated that PIN1-3 and PIN2 may play an important role in root development. In addition, we carried out expression profiling of cotton PIN genes in response to different hormonal treatments and abiotic stresses. The results showed that the majority of cotton PIN genes contained auxin response elements (AuxREs) and salicylic acid (SA) responsive elements in their promoter regions, which were significantly up-regulated by exogenous hormone treatment.

\section{Methods}

Plant materials and growth conditions

Gossypium hirsutum (Xuzhou 142) and Gossypium arboreum (Shixiya 1) seeds were acquired from the Institute of Cotton Research of the Chinese Academy of Agricultural Sciences (Anyang, China). The seeds were planted into sand containers (one seedling per container) and grown in a climate-controlled greenhouse (16-h light and 8-h dark cycle at $30{ }^{\circ} \mathrm{C}$ ) located at Shaanxi Normal University. For each assay, samples were collected from the equivalent growth stages of G. hirsutum and G. arboreum. A total of 9 seedlings of each species were used for each treatment and three biological triplicates were performed per assay.

\section{Identification and phylogenetic analysis of cotton PIN genes}

Genome sequences of G. hisutum, G. arboreum and G. barbadense have recently become available [32-38]. A genome browser for the cotton genome, termed Cottongen, is accessible online (https://www.cottongen.org). The $A$. thaliana genome sequence was acquired from TAIR 10 (http://www.arabidopsis.org). Putative cotton PINs were identified by blast searches against the three reference genomes using $A$. thaliana PIN protein sequences as queries. Next, candidate PINs were further filtered based on their conserved domains using SMART (http://smart.emblheidelberg.de) and Pfam (http://pfam.xfam.org/search\#tabview=tab0) database analyses $[39,40]$. The phylogenetic relationships between putative cotton PINs and $A$. thaliana PIN proteins were determined using the neighbor-joining algorithm using default parameters with 1000 bootstrap analyses by MEGA 5.0 (https://www.megasoftware.net). Based on the phylogenetic analysis, the putative cotton PINs were named 
according to their respective clades. Multiple sequence alignments of all newly identified PINs in this study were performed using ClustalX with default parameters [41].

\section{Chromosomal location and gene duplication analysis}

Chromosome position information of the putative PINs in G. hirsutum was obtained from gene annotation files downloaded from the CottonGen website (https://www.cot tongen.org). The map of PIN gene distribution along the chromosomes is shown from top to bottom [42]. Duplication analysis of G. hirsutum PIN genes and genomic synteny was identified and displayed with the Synteny Mapping and Analysis Program (SyMAP) v3.4 [43].

\section{Stress and hormonal treatments}

Plants used for stress and hormone treatments were grown under the same greenhouse conditions as reported previously [44]. The same protocols were used for drought, dehydration and salt $(250 \mathrm{mM})$ abiotic stress treatments [44]. For the plant hormone treatments, the methods were modified from the methods utilized by Chai et al. [45]. Briefly, two-week old seedlings were irrigated and sprayed with $10 \mu \mathrm{M}$ NAA and $0.5 \mathrm{mM}$ salicylic acid, respectively. After treatment, root and shoot tissues were harvested at indicated times $(0.5,1,3$ and $5 \mathrm{~h})$. The samples were frozen in liquid nitrogen immediately after collection and kept at $-80{ }^{\circ} \mathrm{C}$. For each treatment, three individual samples were collected and analysis was performed on biological triplicates.

\section{RNA extraction and quantitative RT-PCR (qRT-PCR) analysis}

Cotton samples were ground to fine powder with a mortar and pestle in liquid nitrogen. Total RNA was isolated using a modified CTAB method as described [46], and $5 \mu \mathrm{g}$ of total RNA was converted into cDNA using the cDNA Synthesis SuperMix (Transgen, China) according to the manufacture instructions. For qRT-PCR experiments, cotton UBQ7 (GenBank No. AY189972) was used as an internal control. Primers for qRT-PCR analysis are listed in Additional files 1: Table S4.

\section{Analysis of regulatory elements in the promoter region}

Identified GhPIN genes including their predicted promoter sequences were downloaded from the CottonGen website (https://www.cottongen.org). The regulatory elements in the promoter regions were predicted using PLACE and PlantCARE software as previously $[47,48]$.

\section{Accession numbers}

The TAIR accession numbers for the Arabidopsis PIN sequences used in this study are as follows: At1g73590 (AtPIN1), At5g57090 (AtPIN2), At1g70940 (AtPIN3),

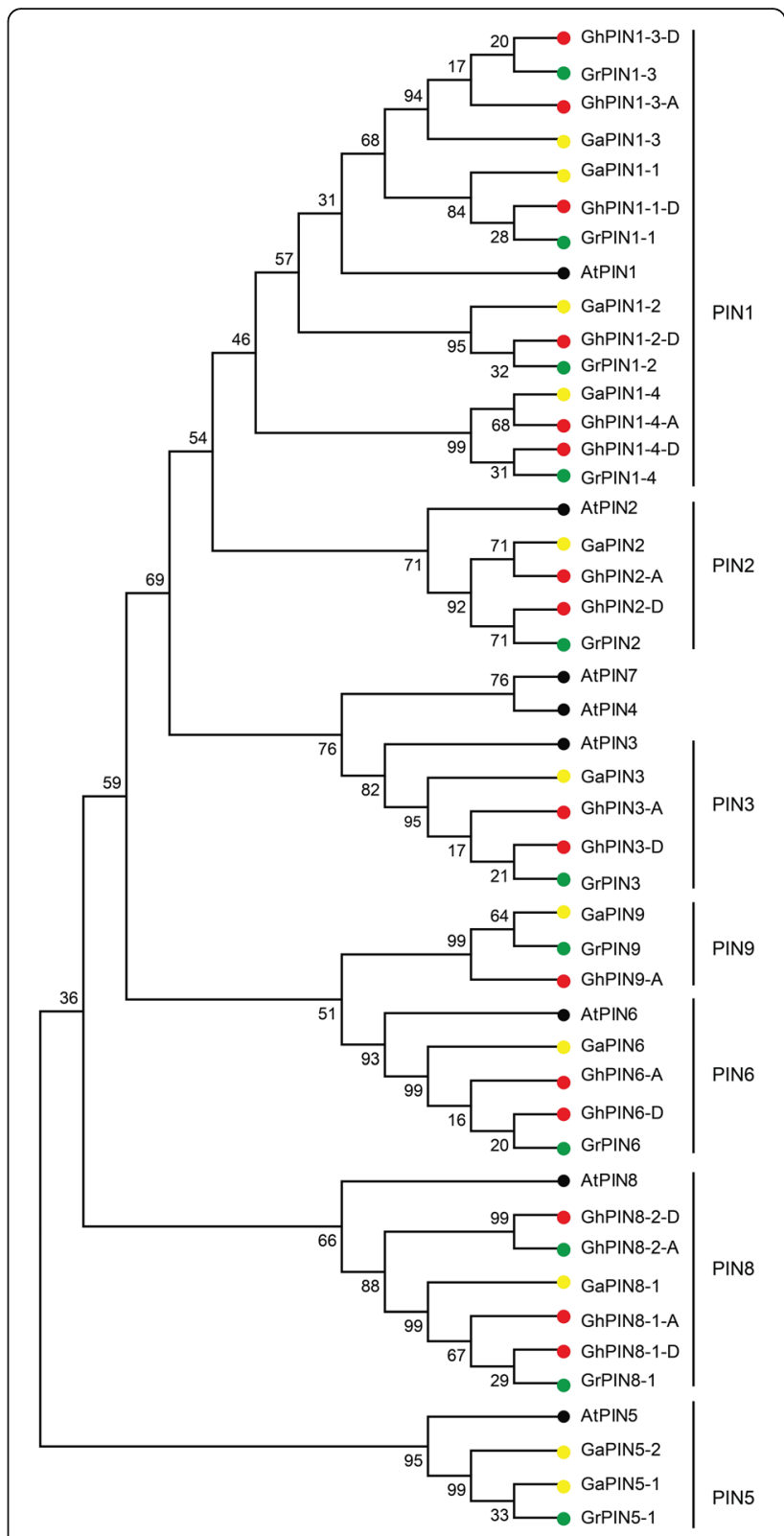

Fig. 1 Phylogenetic analysis of PIN family genes from G. hirsutum, G. arboreum, G. raimondii and A. thaliana. The phylogenetic tree was constructed using MEGA 5.0 with the neighbor-joining method. The origin of the PIN genes are indicated by colored circles as follows: G. hirsutum (red), G. arboretum (yellow), G. raimondii (green), and A. thaliana (black). Numbers on branches are bootstrap values calculated from 1000 replicates

At2g01420 (AtPIN4), At5g16530 (AtPIN5), At1g77110 (AtPIN6), At1g23080 (AtPIN7), At5g15100 (AtPIN8).

\section{Results}

Genome-wide identification of PIN proteins in cotton Three cotton genomes, including two diploid (G. arboreum and G. raimondii) and one allotetraploid (G. hirsutum), have recently been completed and published [33-36]. A genome 
browser is available at the Cottongen website (https:// www.cottongen.org). Putative cotton PIN proteins were initially identified by Blastp searches against the three reference genomes using $A$. thaliana PIN protein sequences as queries. After protein conserved domain and gene structure selection, a total of 17,12 , and 10 predicted protein coding sequences (CDSs) were identified in G. hirsutum, G. arboreum and G. raimondii, respectively (Additional file 2: Table S1). Most G. hirsutum PIN genes contained at least 3 introns in their open reading frames, except PIN1-4-D and PIN8-2-A, which have only one intron and two introns, respectively. The protein coding sequences of all GhPINs are listed in Additional file 3: Table S2. The deduced GhPIN proteins varied in length from 127 amino acids (GhPIN8-2A) to 678 amino acids (GhPIN2-D). Conserved domain analysis showed they have an auxin efflux carrier (ACE) domain at the amino-terminal region and a membrane transport (MT) domain at the carboxy-terminus (Additional file 4: Figure S1). Although the predicted sizes of the putative
GhPIN proteins varied markedly, these two domains were found to be consistently present in all analyzed sequences, indicating that these two domains are likely to be crucial for biochemical function.

\section{Phylogenetic analysis and chromosomal distribution of GhPIN genes}

In order to investigate the evolutionary relationships of the identified PIN proteins, an unrooted phylogenetic tree was generated using the predicted full length amino acid sequences from G. arboreum, G. raimondii, G. hirsutum and A. thaliana. As illustrated in the Neiboring-Joining phylogenetic tree (Fig. 1), a similar organization for the cotton and A. thaliana PIN proteins and some orthologous relationships between both species were identified. Based on this analysis, cotton PIN proteins were named based on their relationships to known A. thaliana PINs. Compared with $A$. thaliana, the PIN1 subfamily was found to be extensively expanded in cotton, indicating they may play an

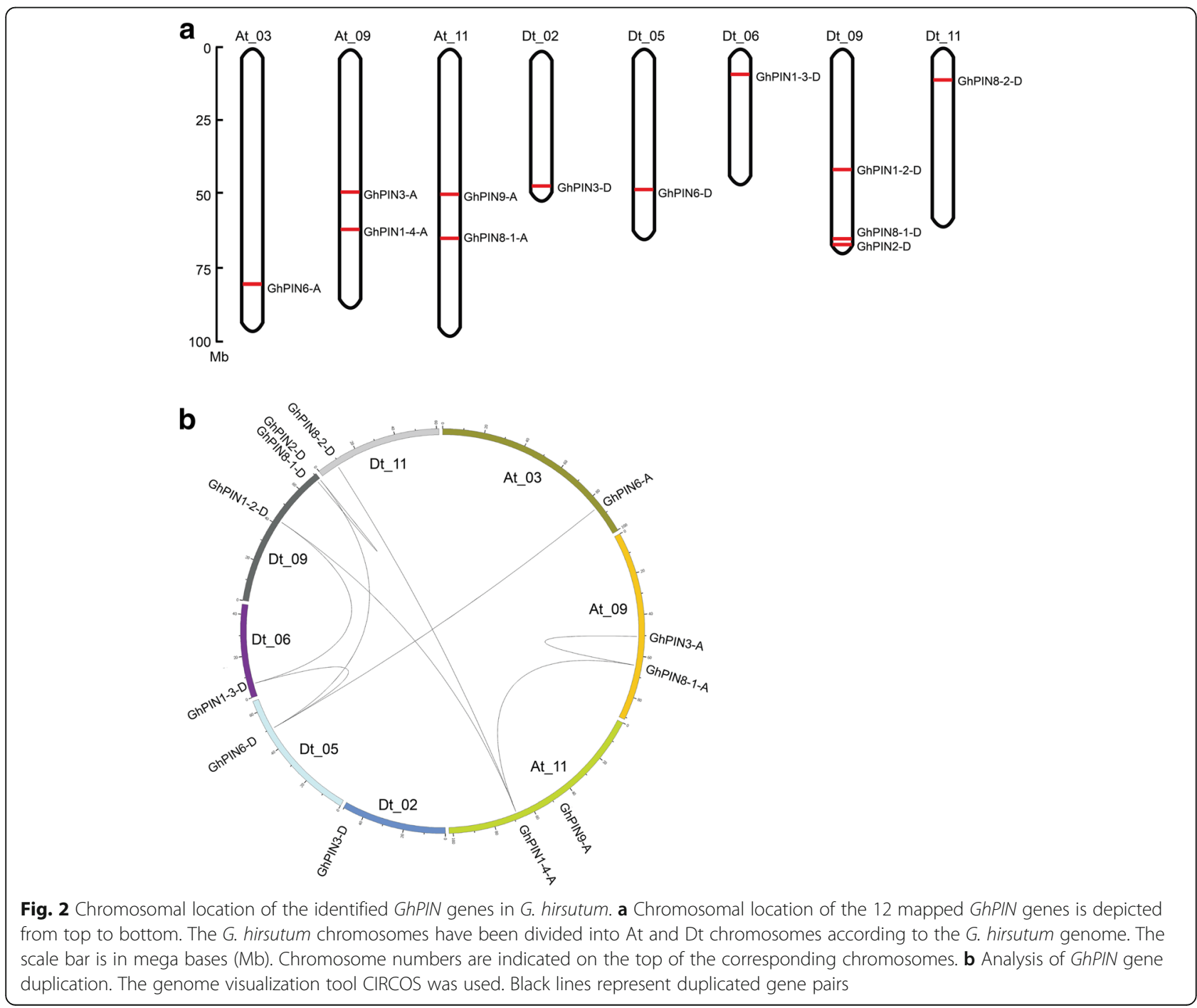


important role in cotton. Interestingly, PIN5 subfamily genes were found in G. arboreum, G. raimondii and $A$. thaliana, but all of them were lost in G. hirsutum and $G$. barbadense (Additional file 5: Table S3), implying that a gene loss event occurred in the PIN family genes after polyploidization in the allotetraploid cotton.

GhPIN genes were mapped onto chromosomes to provide insights into their organization in the cotton genome. This data indicated that 12 genes were distributed among 8 chromosomes, including 5 GhPIN genes on 3 chromosomes from the At-subgenome and 7 genes found on 5 chromosomes from the Dt-subgenome (Fig. 2a). Two whole-genome duplication (WGD) events have been observed in cotton [36], which resulted in the occurrence of gene duplication. The relationships and duplication events between the 12 PIN genes were investigated. These combined data suggested that segmental duplication and dispersed duplication might be the main types of PIN gene duplication in cotton (Fig. 2b).

\section{GhPIN1-3 and GhPIN2 are required for cotton root development}

Roots play an essential role in plant growth by absorbing water and inorganic nutrients from the ground. Auxin, which is transported and regulated by auxin efflux transporters, has been reported as a positional cue for root cell type determination [49]. To evaluate the roles of PIN genes in cotton root development, a comparative analysis of root growth between G. hirsutum and $G$. arboretum was carried out. 7 days after germination, a significant difference in root length was observed between G. hirsutum and G. arboreum (Fig. 3a). The roots of G. hirsutum reached an average length of $8 \mathrm{~cm}$, while roots of G. arboreum were less than $4 \mathrm{~cm}$ (Fig. 3b). Next, we tested the expression of GhPIN genes during root development. qRT-PCR results showed that the expression levels of GhPIN1-3 and GhPIN2 in G. hirsutum were much higher than their predicted orthologs in G. arboreum (Fig. 3c), indicating that GhPIN1-3 and GhPIN2 genes may be required for early root development. After 3 weeks, both the main and lateral roots of $G$. hirsutum were significantly longer than those of G. arboreum (Fig. 4a and b). However, no difference in the number of lateral roots was observed between G. hirsutum and G. arboreum (Additional file 6: Figure S2). Among the 7 PIN genes analyzed, GhPIN1-3 and GhPIN2 were found to be higher expression in both main and lateral roots of $G$. hirsutum relative to G. arboreum (Fig. 4c and d), indicating that GhPIN1-3 and GhPIN2 may play key roles in regulating the latestage development of cotton roots. Furthermore, the expression data of GhPIN2 suggests that it may contribute more than GhPIN1-3 in regulating cotton root development (Figs. 3c and 4c). However, GhPIN1-3 was found to be more highly expressed in lateral roots, suggesting that

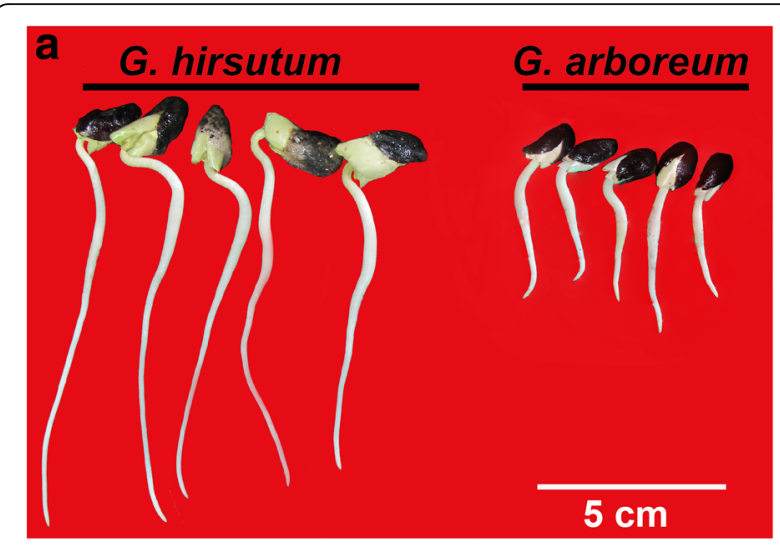

b

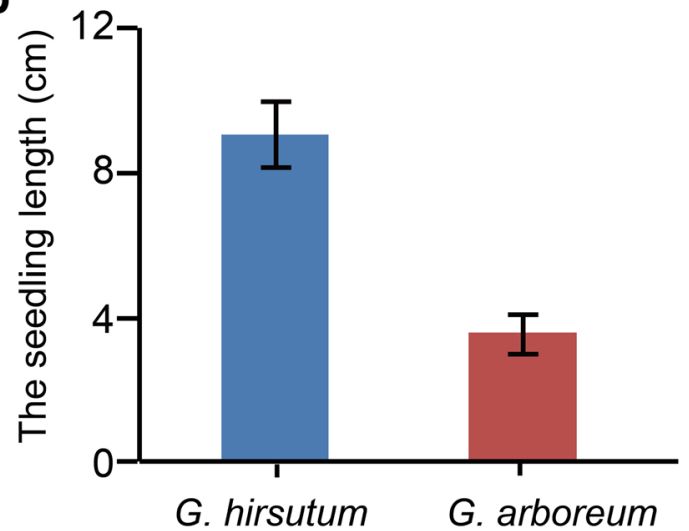

C

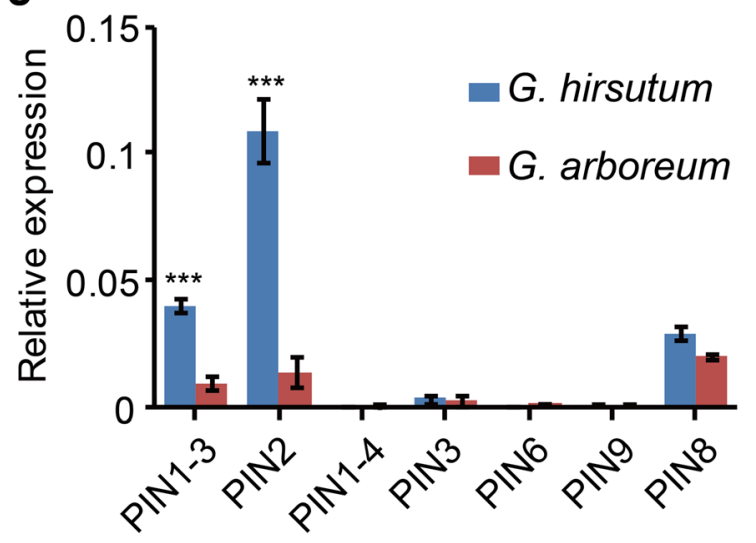

Fig. 3 Comparative analysis of root growth in G. hirsutum and $G$. arboreum. a Representative roots from 7-d-old G. hirsutum and $G$. arboreum seedlings. Bar $=5 \mathrm{~cm}$. b Root lengths of 7-d-old G. hirsutum and $G$. arboreum seedlings as shown in (a). Statistical analysis was carried out on three independent experiments. A total of 10 seedlings were used for each measurement. Root lengths are shown as means \pm SE. c Quantitative RT-PCR analysis of PIN genes in 7-d-old G. hirsutum and $\mathrm{G}$. arboreum roots

it plays more an important role in regulating lateral root growth (Fig. 4d). The observed differences in gene expression during root development indicate possible functional differentiation of PIN genes. 


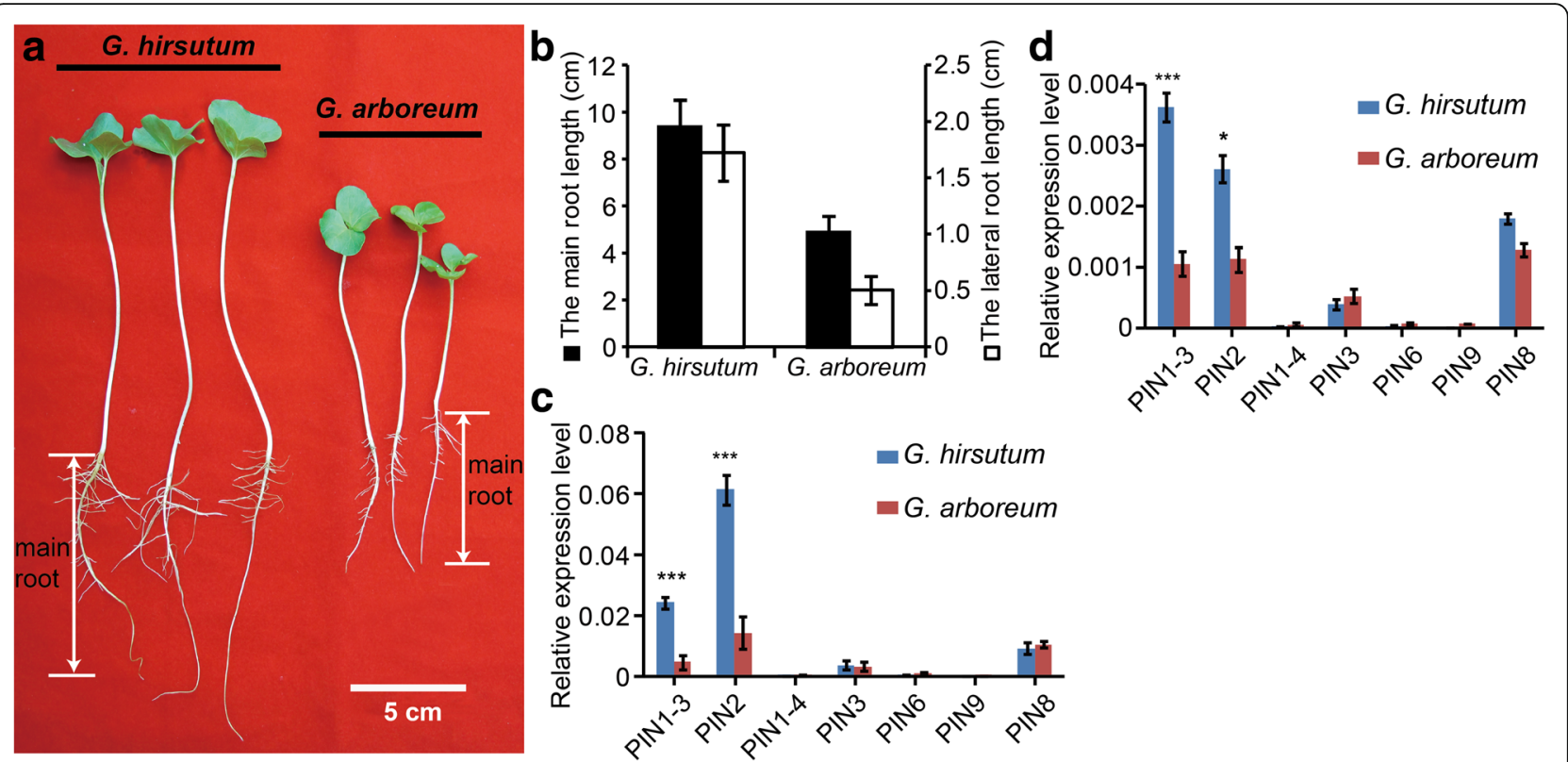

Fig. 4 The development of main and lateral roots in G. hirsutum and G. arboreum. a Comparisons of main and lateral root lengths of three-week-old G. hirsutum and G. arboreum seedlings. Bar $=5 \mathrm{~cm}$. b Measurements of main and lateral root lengths of $G$. hirsutum and $G$. arboreum seedlings as shown in (a). Statistical analyses were carried out on three independent experiments. A total of 9 seedlings were used for each measurement. The lateral root length in this figure represents the average lengths of all investigated lateral roots. Root lengths are shown as means \pm SE. Closed bars with scales to their left side and open bars with scales to their right side indicate the main root lengths and the lateral root lengths, respectively. $\mathbf{c}$, $\mathbf{d}$ The relative expression of PIN genes in main (c) and lateral roots (d) from three week old plants, qRT-PCR experiments were performed on three biological replicates. Error bars represent means \pm SE from three independent experiments. The relative expression level was determined using cotton UBQ7 as a control

\section{Expression of GhPINs in response to drought, salt and dehydration}

It has been reported that PIN genes are involved in plant response to diverse abiotic stresses, such as drought, salt and dehydration [50]. In order to identify potential roles of individual cotton PIN genes in different abiotic stress responses, quantitative real-time PCR (qRT-PCR) analysis was performed to identify changes in gene expression in leaves and roots of plants that underwent different stress treatments. GhPIN1-1-D and GhPIN1-4-A were found to be highly differentially expressed. More GhPINs were upregulated in both leaves and roots that underwent salt and dehydration treatment, relative to plants subjected to drought stress. After cotton seedlings were grown in drought conditions for $48 \mathrm{~h}, 5$ genes were induced in leaves (list genes) while only GhPIN6- $A$ was up-regulated in roots (Fig. 5a). GhPIN1-1-D, GhPIN1-4-A and GhPIN1-4-D were highly upregulated in leaves under drought stress, but concomitantly down-regulated in roots. More genes showed significant changes in gene expression in leaves, when compared to roots, and almost all genes (13 out of 17) were highly expressed in leaves in plants grown in saline conditions (Fig. 5b). Interestingly, the number of GhPIN genes up-regulated in leaves and roots were nearly identical after drought stress (Fig. 5c). In general, GhPIN genes were widely induced by drought, salt and dehydration stresses, suggesting that GhPINs play a role in abiotic stress responses in cotton.

\section{Gene expression analysis of GhPINs in response to auxin and salicylic acid treatment}

The phytohormone auxin is known to play important roles in plant development [51]. Emerging evidence indicates that salicylic acid (SA), a pivotal signaling molecule involved in plant immune responses, is involved in both local and systemic disease resistance responses [52]. In order to explore the effects of auxin and SA on GhPINs expression, GhPIN gene expression analysis was carried out on shoots and roots after treatment with plant hormones were analyzed. GhPINs were differentially expressed upon treatment with SA and the auxin analogue NAA, The results showed that a total of 8 and 10 GhPIN genes were responsive to NAA treatment in shoots and roots, respectively (Fig. 6a). Further, we have also shown that 9 genes in shoots and 10 genes in roots were responsive to SA (Fig. 6b). Auxin responsive elements (AuxREs) and SA responsive elements (SAREs) have been shown to be involved in response to auxin and SA stimuli, respectively $[53,54]$. In order to build our knowledge regarding how these genes are induced by NAA and SA, a comprehensive analysis of GhPIN promoters was performed. Our results showed that 6 out of 8 highly expressed genes possessed at 

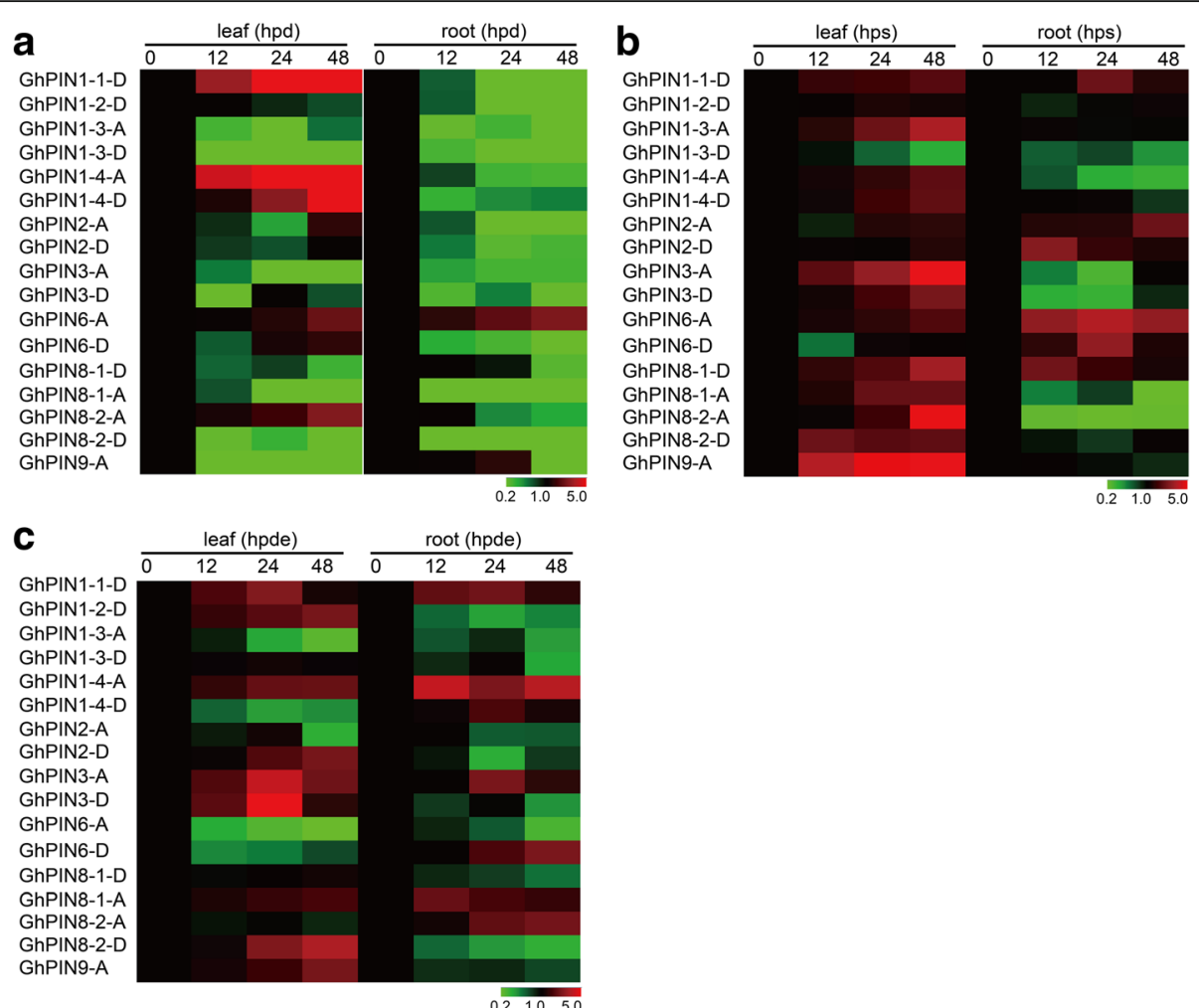

Fig. 5 Gene expression analysis of GhPINs after abiotic stresses treatments. a Fold changes of GhPIN gene expression under drought treatment. b Fold changes of GhPIN gene expression under salt treatment. c Fold changes of GhPIN gene expression under dehydration treatment. All treatments were performed with three biological and three technical replicates. Hpd, hours post drought; hps, hours post salt; hpde, hours post dehydration. The relative gene expression levels were determined using cotton UBQ7 as a control

least one AuxRE in their promoter regions (Fig. 6c). In addition, 4 genes, which were up-regulated in both shoots and roots under SA treatment, contained a predicted SARE in their promoter regions (Fig. 6d).

\section{Discussion}

In this work, 17 PIN genes were identified in G. hirsutum, including 8 that originated from the At subgenome and 9 from the Dt subgenome (Additional file 2: Table S1). Among them, 5 genes from the A genome and 2 from the D genome were lost in the At and Dt subgenomes, which is consistent with previous results that the allotetraploid genome suffered a higher rate of gene loss relative to the diploid genomes, and that more genes were lost in the At subgenome during polyploidization $[34,35]$. Genes in the PIN5 group were observed in G. arboreum and G. raimondii, but not found in G. hirsutum (Fig. 1), suggesting that these genes evolved from a common ancestor, but were then lost in G. hirsutum. Chromosomal distribution of GhPIN genes showed that 12 out of the 17 genes are distributed across 8 chromosomes (Fig. 2a).

Genetic analysis in Arabidopsis has shed light on the function of several PIN genes. Several members of the PIN gene family of auxin efflux carriers, such as AtPIN1,
AtPIN2, AtPIN3, AtPIN4, and AtPIN7, are well-known to be involved in cell-to-cell auxin transport in the root [55]. In particular, AtPIN1 and AtPIN3 were found to be the major auxin transport facilitators mediating polar auxin re-allocation from the shoot to the root tip $[12,55,56]$. The two PIN genes showed high expression in root, thereby determining meristem size and consequently growth rates of the primary root $[56,57]$. In this study, we found that both main and lateral roots in G. hirsutum were longer than those from its diploid ancestor (Figs. 3a and 4a). qRT-PCR analysis revealed that GhPIN1-3 and GhPIN2 were are strongly expressed in both main and lateral roots of $G$. hirsutum (Fig. 4c). The differential and high-level expression of these genes seems to contribute to increased root length in G. hirsutum. Lodging, referred to as the permanent displacement of aboveground parts, greatly depends on root length and strength [15]. Lodging is a common phenomenon that causes yield reductions and makes plants difficult to harvest. GhPIN1-3 and GhPIN2 are required for cotton root development, which can be further used in breeding programs to selecting genotypes that are lodging-resistance.

Previous results revealed that accumulation of the plant hormone indole 3-acetic acid (IAA) in the epidermis of 


\section{a}

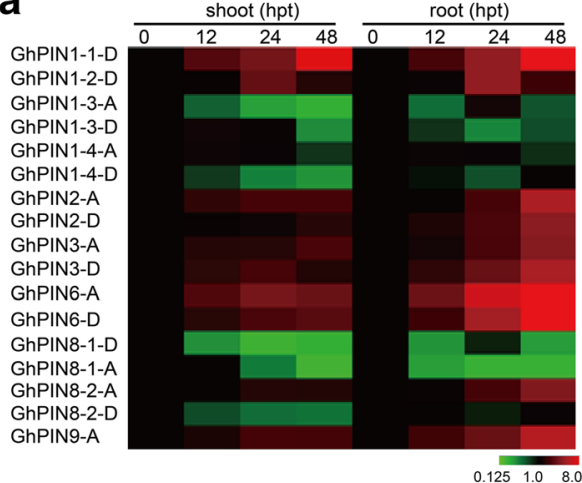

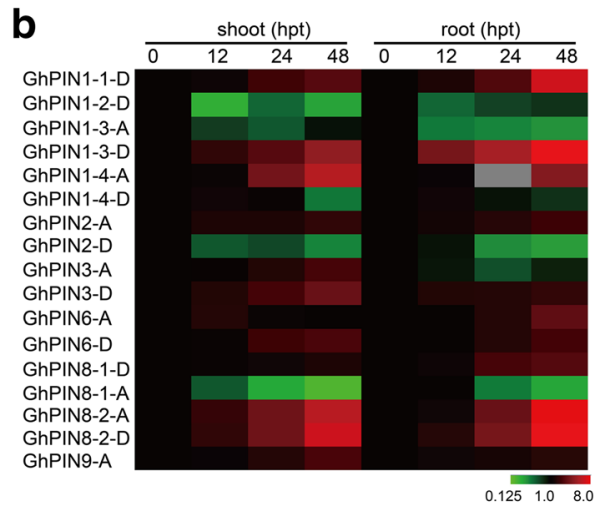


Fig. 6 Quantitative RT-PCR analysis of GhPINs form roots and shoots of cotton plants treated with auxin or salicylic acid. a Expression of GhPIN genes after NAA treatment. b Transcript abundance of GhPIN genes under salicylic acid treatment. Hpt indicates hours post treatment in (a) and (b). c Analysis of GhPINs with AuxRE elements present in their promoter regions. The element (TGTCTC) is the putative ARF binding site, which is one of the key auxin responsive elements (AuxREs). $\mathbf{d}$ Analysis of GhPINs with SA responsive elements present in their promoter regions. The element (TGACG) is the putative SA responsive element

cotton ovules significantly increased the number of lint fibers, an important component of fiber yield [29]. In Arabidopsis, mutation of genes involved in auxin polar transport facilitators resulted in defects in distal organization of roots [39]. Our results showed that GhPIN1-3 and GhPIN2 genes might play an important role in regulating both main and lateral root development (Figs. 3 and 4). Auxin may function in both the promotion of both fiber initiation and cotton root growth, implying that auxin may play a dual functional role in cotton development.

Soybean PIN genes were previously shown to be induced by various abiotic stresses and plant hormones [19]. PIN genes in Sorghum bicolor were also found to be differentially up-regulated after phytohormone treatment and under abiotic stress [58]. Additionally, auxin transporter gene families in maize were reported to be responsive to different abiotic stresses [15]. In the present study, several PIN genes were shown to be highly expressed in cotton plants grown under drought, salt and dehydration treatments (Fig. 5). Further gene-specific overexpression or analysis of PIN knockout plants may be helpful to unravel their functions. 10 out of 17 PINs expressed in cotton roots were induced by NAA and SA treatment (Fig. 6a and b). When analyzing the promoter regions of the 10 PIN genes upregulated by hormone treatment, 6 and 4 were found to contain AuxREs and SAREs in their promoter regions, respectively (Fig. $6 \mathrm{c}$ and d), indicating these elements are very important for PIN genes to respond to NAA and SA stimuli. These findings provide clues towards the identification of more candidates with potential roles in phytohormone stimuli.

\section{Conclusions}

Our study provided a comprehensive analysis of the PIN gene family in G. hirsutum. We showed that PIN1-3 and PIN2 are involved in cotton root development. This study will help us to elucidate the precise role of PIN genes in cotton root development and in adaption to abiotic stress. Our findings will also further help breeding efforts to develop and select the lodging-resistant varieties in the future.

\section{Additional files}

Additional file 1: Table S4. Primers for qRT-PCR experiments. (PDF $52 \mathrm{~kb}$ ) Additional file 2: Table S1. Analysis of G. hirsutum PIN genes and their corresponding orthologues in the AA and DD genomes. (PDF $16 \mathrm{~kb}$ )

Additional file 3: Table S2. The coding sequences of 17 GhPIN genes identified in G. hirsutum. (PDF $31 \mathrm{~kb}$ )

Additional file 4: Figure S1. Multiple sequence alignment of the deduced amino acid sequences of predicted PINs from G. hirsutum. (PDF $2431 \mathrm{~kb}$ )

Additional file 5: Table S3. The number of PIN genes identified across 7 plant species. (PDF $89 \mathrm{~kb}$ )

Additional file 6: Figure S2. Measurements of lateral root number of three-week-old G. hirsutum and G. arboreum seedlings. (PDF 100 kb) 


\section{Abbreviations}

ARF: Auxin response factor; AuxRE: Auxin-responsive element; CTAB: Hexadecyl trimethyl ammonium Bromide; IAA: indole-3-acetic acid; NAA: 1-Naphthaleneacetic acid; SA: Salicylic acid; UBQ: Ubiquinone; WGD: whole-genome duplication

\section{Acknowledgements}

Not applicable.

\section{Funding}

This work was supported by the National Natural Science Foundation of China (Grant No. 31600223), the Fundamental Research Funds for the Central Universities (Grant No. GK201603066 to G.H.X, GK201603066 to H.X and GK201403007 to J.N.Y), and the State Key Laboratory of Cotton Biology Open Fund (Grant No. CB2016A02).

\section{Availability of data and materials}

The cotton genome data analyzed during this article is available from CottonGen (https://www.cottongen.org) and the A. thaliana genome sequence is accessible at TAIR 10 (http://www.arabidopsis.org). The nucleotide sequences of all PIN genes in G. hirsutum can be found in the Additional file 3: Table S2.

\section{Authors' contributions}

GHX and JNY designed the experiment; GHX and PH performed experiments and supervised data analysis; PH, PZ and LMW analyzed data, YZZ and XSW wrote the manuscript. JNY and $H X$ revised the manuscript. All authors contributed to the research and read and approved the final manuscript.

\section{Ethics approval and consent to participate}

All the cotton lines used and analyzed for this study were collected from the Institute of Cotton Research of the Chinese Academy of Agricultural Sciences, which were public and available for non-commercial purpose. This article did not contain any studies with human participants or animals performed by any of the authors.

\section{Consent for publication}

Not applicable.

\section{Competing interests}

The authors declare that they have no competing interests.

\section{Publisher's Note}

Springer Nature remains neutral with regard to jurisdictional claims in published maps and institutional affiliations.

\section{Author details}

${ }^{1}$ Key Laboratory of the Ministry of Education for Medicinal Plant Resources and Natural Pharmaceutical Chemistry, National Engineering Laboratory for Resource Development of Endangered Crude Drugs in the Northwest of China, College of Life Sciences, Shaanxi Normal University, Xi'an 710119, China. ${ }^{2}$ College of Life Sciences, Shaanxi Normal University, Xi'an 710119, China. ${ }^{3}$ National Key Lab of Crop Genetic Improvement, National Center of Crop Molecular Breeding Technology, National Center of Oil Crop Improvement (Wuhan), College of Plant Science and Technology, Huazhong Agricultural University, Wuhan 430070, China.

Received: 19 March 2017 Accepted: 22 June 2017

Published online: 03 July 2017

\section{References}

1. Benjamins R, Scheres B. Auxin: the looping star in plant development. Annu Rev Plant Biol. 2008:59:443-65.

2. Vanneste S, Friml J. Auxin: a trigger for change in plant development. Cell. 2009:136(6):1005-16

3. Chapman EJ, Estelle M. Mechanism of auxin-regulated gene expression in plants. Annu Rev Genet. 2009:43:265-85.

4. Robert HS, Grunewald W, Sauer M, Cannoot B, Soriano M, Swarup R, et al. Plant embryogenesis requires AUX/LAX-mediated auxin influx. Development. 2015;142(4):702-11.
5. Remy E, Cabrito TR, Baster P, Batista RA, Teixeira MC, Friml J, et al. A major facilitator superfamily transporter plays a dual role in polar auxin transport and drought stress tolerance in Arabidopsis. Plant Cell. 2013;25(3):901-26.

6. Vieten A, Sauer M, Brewer PB, Friml J. Molecular and cellular aspects of auxin-transport-mediated development. Trends Plant Sci. 2007;12(4):160-8.

7. Jones AR, Kramer EM, Knox K, Swarup R, Bennett MJ, Lazarus CM, et al. Auxin transport through non-hair cells sustains root-hair development. Nat Cell Biol. 2009;11(1):78-84.

8. Kierzkowski D, Lenhard M, Smith R, Kuhlemeier C. Interaction between meristem tissue layers controls phyllotaxis. Dev Cell. 2013;26(6):616-28.

9. Swarup R, Kargul J, Marchant A, Zadik D, Rahman A, Mills R, et al. Structurefunction analysis of the presumptive Arabidopsis auxin permease AUX1. Plant Cell. 2004;16(11):3069-83.

10. Band LR, Wells DM, Fozard JA, Ghetiu T, French AP, Pound MP, et al. Systems analysis of auxin transport in the Arabidopsis root apex. Plant Cell. 2014;26(3):862-75

11. Petrasek J, Friml J. Auxin transport routes in plant development. Development. 2009;136(16):2675-88.

12. Adamowski M, Friml J. PIN-dependent auxin transport: action, regulation, and evolution. Plant Cell. 2015;27(1):20-32.

13. Peret $B$, Swarup $K$, Ferguson A, Seth M, Yang $Y$, Dhondt $S$, et al. AUX LAX genes encode a family of auxin influx transporters that perform distinct functions during Arabidopsis development. Plant Cell. 2012;24(7): 2874-85.

14. Barbez E, Kubes M, Rolcik J, Beziat C, Pencik A, Wang B, et al. A novel putative auxin carrier family regulates intracellular auxin homeostasis in plants. Nature. 2012;485(7396):119-22.

15. Yue R, Tie S, Sun T, Zhang L, Yang Y, Qi J, et al. Genome-wide identification and expression profiling analysis of ZmPIN, ZmPILS, ZmLAX and ZmABCB auxin transporter gene families in maize (Zea mays L.) under various abiotic stresses. PloS one. 2015;10(3):e0118751.

16. Ding Z, Galvan-Ampudia CS, Demarsy E, Langowski L, Kleine-Vehn J, Fan Y, et al. Light-mediated polarization of the PIN3 auxin transporter for the phototropic response in Arabidopsis. Nat Cell Biol. 2011;13(4):447-52.

17. Kleine-Vehn J, Ding Z, Jones AR, Tasaka M, Morita MT, Friml J. Gravityinduced PIN transcytosis for polarization of auxin fluxes in gravity-sensing root. Proc Natl Acad Sci. 2010;107(51):22344-9.

18. Petrášek J, Mravec J, Bouchard R, Blakeslee JJ, Abas M, Seifertová D, et al. PIN proteins perform a rate-limiting function in cellular auxin efflux. Science. 2006:312(5775):914-8.

19. Wang HZ, Yang KZ, Zou JJ, Zhu LL, Xie ZD, Morita MT, et al. Transcriptional regulation of PIN genes by FOUR LIPS and MYB88 during Arabidopsis root gravitropism. Nat Commun. 2015;6:8822

20. Ding Z, Wang B, Moreno I, Duplakova N, Simon S, Carraro N, et al. ERlocalized auxin transporter PIN8 regulates auxin homeostasis and male gametophyte development in Arabidopsis. Nat Commun. 2012;3:941.

21. Mravec J, Skupa P, Bailly A, Hoyerova K, Krecek P, Bielach A, et al. Subcellular homeostasis of phytohormone auxin is mediated by the ER-localized PIN5 transporter. Nature. 2009:459(7250):1136-40.

22. Guo X, Qin Q, Yan J, Niu Y, Huang B, Guan L, et al. TYPE-ONE PROTEIN PHOSPHATASE4 regulates pavement cell interdigitation by modulating PIN-FORMED1 polarity and trafficking in Arabidopsis. Plant Physiol. 2015; 167(3):1058-75.

23. Alonso-Peral MM, Candela H, Del Pozo JC, Martinez-Laborda A, Ponce MR, Micol JL. The HVE/CAND1 gene is required for the early patterning of leaf venation in Arabidopsis. Development. 2006;133(19):3755-66.

24. Luschnig C, Gaxiola RA, Grisafi P, Fink GR. EIR1, a root-specific protein involved in auxin transport, is required for gravitropism in Arabidopsis thaliana. Genes Dev. 1998;12(14):2175-87.

25. Utsuno K, Shikanai T, Yamada Y, Hashimoto T. Agr, an Agravitropic locus of Arabidopsis thaliana, encodes a novel membrane-protein family member. Plant Cell Physiol. 1998;39(10):1111-8.

26. Müller A, Guan C, Gälweiler L, Tänzler P, Huijser P, Marchant A, et al. AtPIN2 denfines a locus of Arabidopsis for root gravitropism control. EMBO J. 1998; 17(23):6903-11.

27. Wang JR, Hu H, Wang GH, Li J, Chen JY, Wu P. Expression of PIN genes in rice (Oryza sativa L.): tissue specificity and regulation by hormones. Mol Plant. 2009:2(4):823-31.

28. Beasley CA, Egli MA, Chang SR, Radin JW. Independent control of fiber development and nitrate reduction in cultured cotton ovules. Plant physiology. 1979;63(1):57-60. 
29. Zhang M, Zheng X, Song S, Zeng Q, Hou L, Li D, et al. Spatiotemporal manipulation of auxin biosynthesis in cotton ovule epidermal cells enhances fiber yield and quality. Nat Biotechnol. 2011;29(5):453-8.

30. Zhang $M$, Xiao $Y$, Zeng J, Pei Y. PIN-formed protein, a door to reveal the mechanism for auxin-triggered initiation of cotton fiber. Plant Signal Behav. 2017; doi:10.1080/15592324.2017.1319031.

31. Zhang M, Zeng JY, Long $H$, Xiao YH, Yan XY, Pei Y. Auxin regulates cotton fiber initiation via GhPIN-mediated auxin transport. Plant and Cell Physiology, 2016: pcw203. doi: 10.1093/pcp/pcw203.

32. Paterson AH, Wendel JF, Gundlach H, Guo H, Jenkins J, Jin D, et al. Repeated polyploidization of Gossypium genomes and the evolution of spinnable cotton fibres. Nature. 2012;492(7429):423-7.

33. Wang K, Wang Z, Li F, Ye W, Wang J, Song G, et al. The draft genome of a diploid cotton Gossypium raimondii. Nat Genet. 2012:44(10):1098-103.

34. Zhang T, Hu Y, Jiang W, Fang L, Guan X, Chen J, et al. Sequencing of allotetraploid cotton (Gossypium hirsutum L. acc. TM-1) provides a resource for fiber improvement. Nat Biotechnol. 2015;33(5):531-7.

35. Li F, Fan G, Lu C, Xiao G, Zou C, Kohel RJ, et al. Genome sequence of cultivated upland cotton (Gossypium hirsutum TM-1) provides insights into genome evolution. Nat Biotechnol. 2015;33(5):524-30.

36. Li F, Fan G, Wang K, Sun F, Yuan Y, Song G, et al. Genome sequence of the cultivated cotton Gossypium arboreum. Nat Genet. 2014;46(6):567-72.

37. Liu X, Zhao B, Zheng HJ, Hu Y, Lu G, Yang CQ, et al. Gossypium barbadense Genome sequence provides insight into the evolution of extra-long staple fiber and specialized metabolites. Sci Rep. 2015;5:14139.

38. Yuan D, Tang Z, Wang M, Gao W, Tu L, Jin X, et al. The genome sequence of Sea-Island cotton (Gossypium barbadense) provides insights into the allopolyploidization and development of superior spinnable fibres. Sci Rep. 2015:5:17662.

39. Finn RD, Coggill P, Eberhardt RY, Eddy SR, Mistry J, Mitchell AL, et al. The Pfam protein families database: towards a more sustainable future. Nucleic Acids Res. 2016:44(D1):D279-85.

40. Letunic I, Doerks T, Bork P. SMART: recent updates, new developments and status in 2015. Nucleic Acids Res. 2015;43(Database issue):D257-60.

41. Thompson JD, Gibson TJ, Plewniak F, Jeanmougin F, Higgins DG. The CLUSTAL_X windows interface: flexible strategies for multiple sequence alignment aided by quality analysis tools. Nucleic Acids Res. 1997;25(24): 4876-82.

42. Zou C, Lu C, Shang H, Jing X, Cheng H, Zhang Y, et al. Genome-wide analysis of the sus gene family in cotton. J Integr Plant Biol. 2013;55(7):643-53.

43. Soderlund C, Bomhoff M, Nelson WM. SyMAP v3.4: a turnkey synteny system with application to plant genomes. Nucleic acids research. 2011; 39(10):e68.

44. Ji SJ, Lu YC, Feng JX, Wei G, Li J, Shi YH, et al. Isolation and analyses of genes preferentially expressed during early cotton fiber development by subtractive PCR and cDNA array. Nucleic Acids Res. 2003;31(10):2534-43.

45. Chai C, Wang Y, Joshi T, Valliyodan B, Prince S, Michel L, et al. Soybean transcription factor ORFeome associated with drought resistance: a valuable resource to accelerate research on abiotic stress resistance. BMC Genomics. 2015;16:596

46. Jin X, Li Q, Xiao G, Zhu YX. Using genome-referenced expressed sequence tag assembly to analyze the origin and expression patterns of Gossypium hirsutum transcripts. J Integr Plant Biol. 2013;55(7):576-85.

47. Higo K, Ugawa Y, Iwamoto M, Korenaga T. Plant cis-acting regultory DNA elements (PLACE) database. Nucleic Acids Res. 1999:27(1):297-300.

48. Lescot M, Déhais $P$, Thijs $G$, Marchal $K$, Moreau $Y$, Van de Peer $Y$, et al. PlantCARE, a database of plant cis-acting regulatory elements and a portal to tools for in silico analysis of promoter sequences. Nucleic Acids Res. 2002;30(1):325-7.

49. Wang JJ, Guo HS. Cleavage of INDOLE-3-ACETIC ACID INDUCIBLE28 mRNA by microRNA847 upregulates auxin signaling to modulate cell proliferation and lateral organ growth in Arabidopsis. Plant Cell. 2015;27(3):574-90.

50. Habets ME, Offringa R. PIN-driven polar auxin transport in plant developmental plasticity: a key target for environmental and endogenous signals. The New phytologist. 2014;203(2):362-77.

51. Zhao Y. Auxin biosynthesis and its role in plant development. Annu Rev Plant Biol. 2010;61:49-64

52. Shah J. The salicylic acid loop in plant defense. Curr Opin Plant Biol. 2003; 6(4):365-71.

53. Pieterse CM, Van Loon LC. NPR1: the spider in the web of induced resistance signaling pathways. Curr Opin Plant Biol. 2004;7(4):456-64.
54. Ulmasov T, Hagen G, Guilfoyle TJ. ARF1, a transcription factor that binds to auxin response elements. Science. 1997:276(5320):1865-8.

55. Krecek P, Skupa P, Libus J, Naramoto S, Tejos R, Friml J. E. Z. The PIN-FORMED (PIN) protein family of auxin transporters. Genome biology. 2009;10(12):249.

56. Blilou I, Xu J, Wildwater M, Willemsen V, Paponov I, Friml J, et al. B S. The PIN auxin efflux facilitator network controls growth and patterning in Arabidopsis roots. Nature. 2005;433(7021):39-44.

57. Omelyanchuk NA, Kovrizhnykh W, Oshchepkova EA, Pasternak T, Palme K, Mironova W. A detailed expression map of the PIN1 auxin transporter in Arabidopsis thaliana root. BMC plant biology. 2016; 16 Suppl 1:5.

58. Shen C, Bai Y, Wang S, Zhang S, Wu Y, Chen M, et al. Expression profile of PIN, AUX/LAX and PGP auxin transporter gene families in Sorghum bicolor under phytohormone and abiotic stress. FEBS J. 2010;277(14):2954-69.

\section{Submit your next manuscript to BioMed Central and we will help you at every step:}

- We accept pre-submission inquiries

- Our selector tool helps you to find the most relevant journal

- We provide round the clock customer support

- Convenient online submission

- Thorough peer review

- Inclusion in PubMed and all major indexing services

- Maximum visibility for your research

Submit your manuscript at www.biomedcentral.com/submit
) Biomed Central 